\title{
Clear cell carcinoma of the uterine cervix: clinical characteristics and feasibility of fertility-preserving treatment
}

This article was published in the following Dove Press journal:

OncoTargets and Therapy

9 January 2014

Number of times this article has been viewed

\author{
Xiang Jiang \\ Ying Jin \\ Yan Li \\ Hui-Fang Huang \\ Ming Wu \\ Keng Shen \\ Ling-Ya Pan
}

Department of Obstetrics and Gynecology, Peking Union Medical College Hospital, Chinese Academy of Medical Sciences and Peking Union Medical College, Beijing, People's Republic of China
Correspondence: Ying Jin

Department of Obstetrics and Gynecology, Peking Union Medical College Hospital, Chinese Academy of Medical Sciences and Peking Union Medical College, I Shuai Fu Yuan, Wang Fu Jing Street, Beijing 100730, People's Republic of China

Tel +861065296218

Fax $+86 \quad 1065124875$

Email jinypumc@aliyun.com
Abstract: The objective of this retrospective study was to analyze the clinical characteristics and prognosis of clear cell adenocarcinoma (CCA) in the post-diethylstilbestrol (DES) era and to evaluate the feasibility of fertility-preserving treatment. The records of 32 patients with CCAs who were treated at Peking Union Medical College Hospital from August 1986 to June 2012 were retrospectively reviewed. Three of the patients had undergone fertility-preserving treatment. The incidence of CCA among cervical adenocarcinomas was $15.2 \%$. The median age was 38 years: 11 patients $(34.4 \%)$ were diagnosed before 30 years of age and two $(6.3 \%)$ after 70 years of age. Ten patients $(31.2 \%)$ were nulliparous. No patient had been exposed to DES. Twenty-nine patients $(90.6 \%)$ presented with obvious symptoms, and the cervix appeared abnormal in 26 patients (81.3\%). Cervical Papanicolaou (Pap) tests were abnormal in all four patients in whom they were performed (three had high-grade squamous intraepithelial lesions and one had atypical squamous cells of undetermined significance). The distribution by stage was $56.3 \%$ stage I, $34.4 \%$ stage II, $6.3 \%$ stage III, and $3.1 \%$ stage IV. Treatments mainly included surgery for patients with stage I to IIA CCA and radiochemotherapy for patients with advanced CCA. The overall 5-year progression-free survival was $72.2 \%$. Patients with stage I to IIA CCA had better 5-year progression-free survival than did patients with stage IIB to IV CCA $(81.5 \%$ versus $40.0 \%, P=0.003)$. The three patients who had undergone fertility-preserving treatment had no recurrences. CCA may also affect adolescents and children without prior DES exposure, who are often misdiagnosed as having functional uterine bleeding. Radiotherapy appears to be effective for local control but to have no effect on distant recurrences. In our study, the prognosis of patients with early-stage CCA, including those who had undergone fertility-preserving treatment, was not inferior to that of patients with other types of cervical adenocarcinoma.

Keywords: clear cell carcinoma, cervix, diagnosis, prognosis, fertility-preserving

\section{Introduction}

Clear cell adenocarcinoma of the cervix (CCA), which is a relatively rare tumor of the lower genital tract, is characterized by abundant clear cytoplasm and hobnail cells. ${ }^{1,2}$ CCA has reportedly been diagnosed in women with a history of exposure to diethylstilbestrol (DES) in utero. The relationship between intrauterine exposure to DES and CCA was explored in a case-control study in 1971 and further confirmed by subsequent animal experiments and a cohort study designed in the early 1990s. ${ }^{3-5}$ Since the ban on DES in the 1970s, this disease has become extremely rare. According to Reich et al, ${ }^{1}$ CCAs account for $4 \%-9 \%$ of cervical adenocarcinomas in patients who have not been exposed to DES, implying that these tumors can also develop in the absence of such exposure. Current research suggests that many factors, including 
cervical endometriosis, contribute to the occurrence of CCA. ${ }^{6}$ However, it is believed that human papillomavirus is of limited importance in non-DES-related CCA. ${ }^{7-10}$

Because this disease is so rare, many of its aspects remain unclear. One multicenter study that included 34 CCAs from the post-DES era reported that clear cell histology in and of itself does not appear to portend a worse prognosis. ${ }^{11}$ In view of the bimodal age distribution of CCA patients, ${ }^{12}$ the effectiveness of fertility-preserving treatment is a key issue that requires clarification. To date, only a few case reports have implied that fertility-preserving treatment is feasible in patients with early-stage CCA. ${ }^{13}$

The aim of this study was to identify the clinical characteristics, and in particular the prognosis, of CCA patients without a history of DES exposure. The feasibility of fertility-preserving treatment is also discussed.

\section{Patients and methods}

A retrospective review of patients with primary CCA referred to Peking Union Medical College Hospital between 1986 and 2012 was conducted. The diagnoses of all participating patients were confirmed by a pathologist in our hospital. CCA is characterized microscopically by prominent cell borders and clear cytoplasm, sometimes with focal gland formation (Figure 1A and 1B). Medical records, including relevant patient characteristics, pathological findings, treatment, and findings at follow-up, were collected.

Staging was based on the 2009 International Federation of Gynecology and Obstetrics (FIGO) staging system for cervical cancers. ${ }^{14}$

The follow-up schedule involved visits every 3-4 months for the first 2 years, every 6 months in years 3-5, and yearly from then on. At each visit, physical examination, vaginal vault cytology, and radiological and biological investigations were performed. The follow-up period was defined as the time interval between the date of surgery (or diagnosis in nonsurgical patients) and either the date of death or the latest follow-up visit.
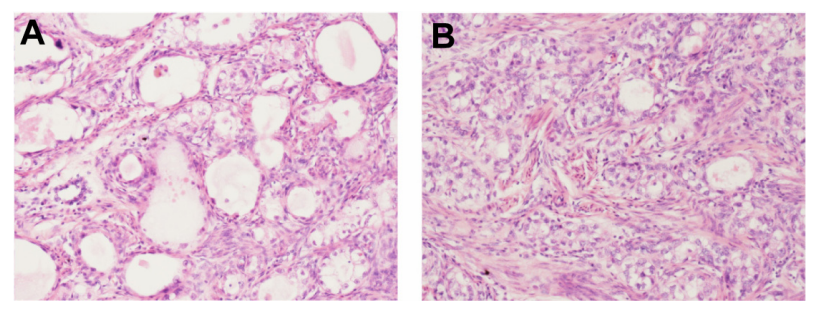

Figure I Microscopic characteristics of clear cell adenosquamous carcinoma of the cervix.

Notes: Magnification $=40 \times$. (A) Sheets of tumor cells with prominent cell borders and clear cytoplasm. (B) Focal areas of gland formation.
The data were analyzed using SPSS 16.0 statistical software (IBM Corporation, Armonk, NY, USA). Survival curves were plotted with the Kaplan-Meier method and tested using the log-rank test. $P<0.05$ was considered statistically significant.

\section{Results}

Thirty-two cases of CCA (15.2\%) were identified among 211 patients with cervical adenocarcinoma. The median age of these patients at the time of diagnosis was 38 years (range, 12-74 years). The age distribution is shown in Figure 2. Eleven patients $(34.4 \%)$ were diagnosed before 30 years of age and two $(6.3 \%)$ were diagnosed after 70 years of age. Ten patients $(31.2 \%)$ were nulliparous. No patient had a history of DES exposure. Twenty-nine patients (90.6\%) presented with obvious symptoms, including abnormal vaginal bleeding $(n=16)$, vaginal discharge $(n=4)$, postcoital bleeding $(n=7)$, abdominal pain $(n=1)$, and blood in the feces $(n=1)$. Among the three asymptomatic patients, one had associated deep venous thrombosis and the other two were diagnosed by routine cervical screening. Twenty-six patients $(81.3 \%)$ had abnormal-looking cervixes; 20 had visible exophytic lesions and six had barrel-shaped cervixes. The remaining six patients had normal-appearing cervixes; two had pelvic masses visible by B-mode ultrasound and four were found to have endocervical lesions in their postoperative specimens. Cervical Papanicolaou (Pap) tests were abnormal in all four patients in whom they were performed (three patients had high-grade squamous intraepithelial lesions and one had atypical squamous cells of undetermined significance). The distribution by stage was: stage I, 56.3\% ( $n=18$; one patient had stage IA2 CCA, 15 had stage IB1 CCA, and

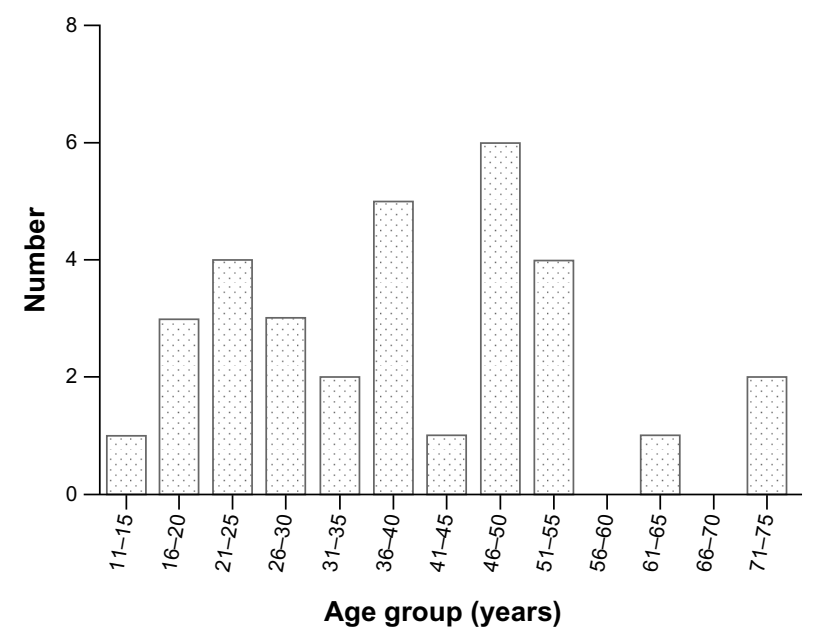

Figure 2 Age distribution (years) of patients with CCA. Abbreviation: CCA, clear cell adenocarcinoma. 
two patients had stage IB2 CCA); stage II, 34.4\% ( $n=11$; four patients had stage IIA1 CCA, three had stage IIA2 CCA, and four had stage IIB); stage III, $6.3 \%(n=2$; one patient had stage IIIA CCA and one had stage IIIB CCA); and stage IV, $3.1 \%$ (one patient with stage IVB CCA). Median follow-up time was 35 months (range, 2-128 months).

Among 25 FIGO stage IA2-IIA2 patients, 22 underwent surgery: 19 underwent radical hysterectomy, and three underwent fertility-preserving treatment (two cervical trachelectomy and one cervical conization). Pelvic lymphadenectomy was performed in 21 patients, yielding a median of 19 nodes (range, 6-46 nodes). Para-aortic lymphadenectomy was performed in three patients $(13.0 \%)$, yielding a median of two nodes (range, 2-4 nodes). Pathological examination showed pelvic lymph node involvement in two patients $(9.5 \%)$, deep cervical stromal invasion in seven patients (31.8\%), and lymph-vascular space involvement in three patients $(13.6 \%)$. The characteristics of the three patients who underwent fertility-preserving surgery are shown in Table 1. The remaining three patients received chemoradiation. Figure 3 shows positron emission tomography-computed tomography imaging of changes in cervical carcinomas before and after treatment.

Seven patients (two with stage IB1 CCA, two with stage IB2 CCA, two with stage IIA1 CCA, and one with stage IIA2 $\mathrm{CCA}$ ) received neoadjuvant chemotherapy before surgery. Postoperative adjuvant radiotherapy was administered to ten patients with adverse risk factors; seven of these patients also received concurrent chemotherapy. Two patients received postoperative chemotherapy. The chemotherapy regimens were platinum plus 5-fluorouracil or carboplatin plus paclitaxel. Treatment of patients with advanced CCA (four with stage IIB CCA, two with stage III CCA, and one with stage IVB CCA) consisted of chemoradiation ( $\mathrm{n}=3$ ), radiation plus extrafascial hysterectomy $(\mathrm{n}=1)$, and supportive treatment $(\mathrm{n}=1$ patient with stage IVB CCA who had an intestinal obstruction). Two of these patients died after two courses of chemotherapy.

The 5-year progression free survival (PFS) rate among all 32 patients was $72.2 \%$. As expected, patients with early stage (I-IIA) CCA had better 5-year PFS than those with advanced stages (IIB-IVB) $(81.5 \%$ versus $40.0 \%, P=0.003)$ (Figure 4 ).
Tumor size had no impact on PFS. The characteristics of four patients with uncontrolled or recurrent disease are shown in Table 2. The three patients who underwent fertility-preserving surgery remained disease free after follow-ups of 8 months, 16 months, and 54 months.

\section{Discussion}

CCA is a rare disease, especially since the banning of DES. In this study, the prevalence of CCA was 32 (15.2\%) of all 211 adenocarcinomas of the cervix, which is higher than the previously reported $4 \%-9 \%,{ }^{1}$ perhaps because all our patients had been referred to a specialized and prestigious gynecology treatment center. The largest previously published study of CCA included only 34 cases of CCA from multiple centers. ${ }^{11}$ In the present study, we examined the records of 32 cases of CCA to accumulate more data on CCA patients with no history of DES exposure.

CCAs tend to occur in pediatric and adolescent patients who were exposed in utero to DES; whether this is also true of patients without DES exposure in utero is uncertain. According to analysis of the records of 41 patients without prior DES exposure in the central registry of the Netherlands, non-DES-associated CCAs still have a bimodal age distribution, with one peak at 26 years and a second peak at 71 years. ${ }^{12}$ However, Thomas et al, ${ }^{11}$ reporting on a cohort of CCA patients that included only $6 \%$ with confirmed DES exposure, stated that CCAs tend to affect older women (median age 53 years). In the present study, the median age at the time of diagnosis was 38 years; $34.4 \%$ of patients were diagnosed before 30 years of age. Our study suggests that, even in the post-DES era, CCA tends to affect adolescents and young women more than other types of cervical adenocarcinoma do. Despite the small sample size, we did identify a bimodal distribution.

As a type of adenocarcinoma of the cervix, CCA can be difficult to diagnose accurately. Whereas a timely diagnosis is often made by cervical biopsy in adult patients, CCA in young virginal patients is often misdiagnosed as functional uterine bleeding, resulting in a delay in the correct diagnosis until a large mass that is obvious on imaging has grown. ${ }^{15}$ In our series, one 19-year-old patient had presented with

Table I Characteristics of patients who underwent fertility-preserving treatment $(n=3)$

\begin{tabular}{lllllll}
\hline Patient & Age (years) & Stage & Tumor diameter $(\mathbf{c m})$ & Treatment & Pathological results & Result \\
\hline I & 28 & IA2 & 0.5 & LND+VRT & No residual disease* \\
2 & 25 & IBI & I.5 & LND+ART+chemotherapy & Superficial stromal invasion & No rec (8 months) \\
3 & 20 & IBI & I.5 & Conization+chemotherapy & No residual disease* & No rec (I6 months) \\
\hline
\end{tabular}

Note: *After preoperative diagnostic loop electrosurgical excision and biopsy of cervical neoplasm.

Abbreviations: n, number; LND, lymphadenectomy; VRT, vaginal radical trachelectomy; rec, recurrence; ART, transabdominal radical trachelectomy. 

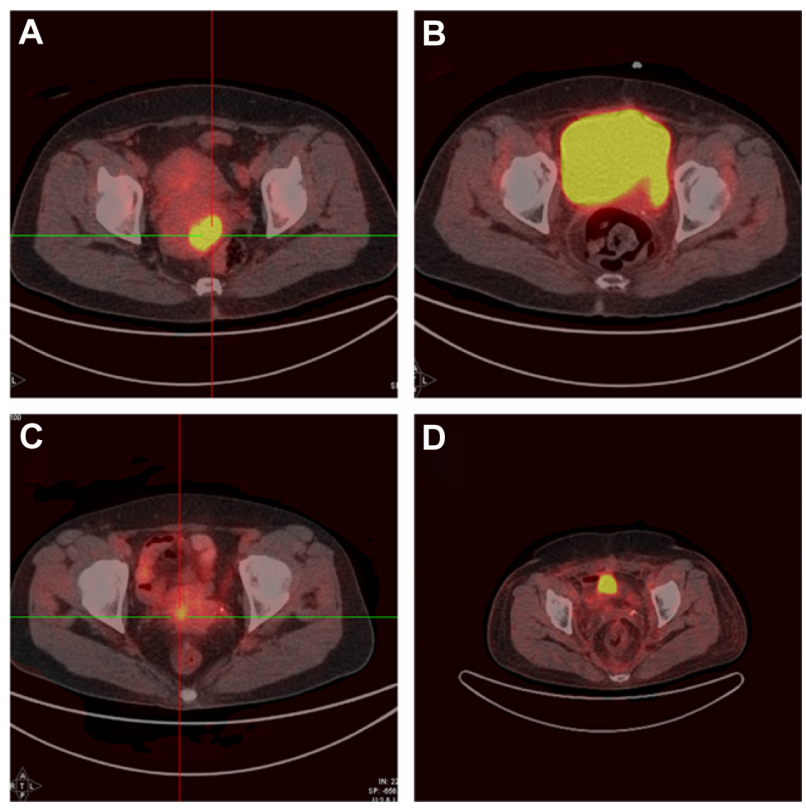

Figure 3 PET-CT images of cervical carcinomas of patients with CCA showing changes before and after treatment.

Notes: (A) PET-CT image of Case I before treatment. (B) PET-CT image of Case I after treatment. (C) PET-CT image of Case 2 before treatment. (D) PET-CT image of Case 2 after treatment.

Abbreviations: PET-CT, positron emission tomography-computed tomography; CCA, clear cell adenocarcinoma.

abnormal vaginal bleeding; B-mode ultrasound had shown a pelvic mass and the diagnosis was eventually made by exploratory laparotomy. A previous report found that most CCA lesions are endophytic and prone to deep infiltration. ${ }^{1}$ Such lesions are often not detected during gynecological examinations or by Pap tests. Thomas et $\mathrm{al}^{11}$ reported that only six of 31 CCA patients $(18 \%)$ had abnormal Pap tests. ${ }^{11}$ In our series, because most patients presented with obvious symptoms and $26(81.3 \%)$ had abnormal-looking cervixes,

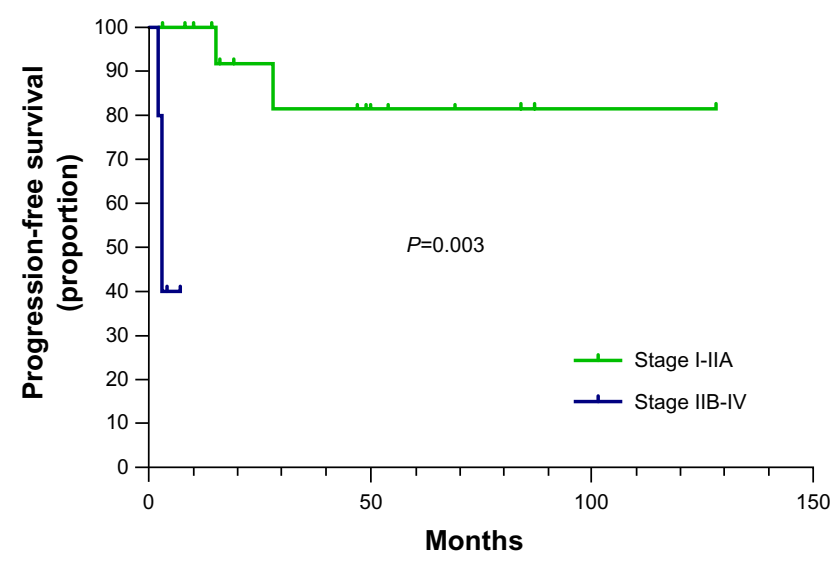

Figure 4 Kaplan-Meier plot of PFS in stage I-IIA CCA versus stage IIB-IV CCA. Note: The 5-year PFS of patients with stage I-IIA CCA is significantly better than that of those with stage IIB-IVB CCA (8I.5\% versus $40.0 \%, P=0.003)$.

Abbreviations: PFS, progression-free survival; CCA, clear cell adenocarcinoma. there was a relatively high proportion of early-stage (FIGO stage I-IIA2) diagnoses (78.1\%, 25 of 32 patients).

Owing to the rarity of CCA, current treatment is based mainly on experience with non-CCA adenocarcinomas and squamous cell carcinomas (SCCs). On the basis of a systematic review, Baalbergen et al concluded that, except for those in whom lymph involvement is implied by imaging findings, early-stage adenocarcinoma patients benefit more from radical hysterectomy than from chemoradiotherapy. ${ }^{16}$ Results from a Phase III randomized trial indicated that radiotherapy was particularly beneficial for high-risk patients with adenocarcinoma or adenosquamous histology. ${ }^{17}$ Although radiotherapy appeared to be effective for local control, it had no effect on distant recurrence, which was best treated by systematic chemotherapy. The findings in our study were similar. Those who received only postoperative radiation appeared to have a high risk for distant recurrence (Table 2). Whether adjuvant radiation plus systematic chemotherapy can reduce the relapse rate in high-risk CCA patients is still uncertain.

Although clear cell ovarian carcinomas have an unfavorable prognosis, clear cell cervical cancer in and of itself does not have a worse prognosis than SCC, according to Thomas et al. ${ }^{11}$ Reich et al ${ }^{1}$ reported no statistically significant differences between the 5-year survival rates of 15 patients with CCA, 444 with SCC, and 59 with non-clear cell adenocarcinomas. However, the authors of one large study with a multivariable analysis of the data of 4,103 patients with adenocarcinoma reported that adenocarcinoma histology negatively impacts survival of patients with early-stage and advanced-stage carcinomas and suggested that histology should be taken into account in treatment planning. ${ }^{18}$ Univariate analysis had previously identified both advanced stage and pelvic lymph node involvement as prognostic factors in CCA. Patients with stage I to IIA disease reportedly have a better 3-year overall survival than do patients with advanced-stage disease (91\% versus $22 \%$, respectively; $P=0.001)$. In addition, the presence of positive lymph nodes has a negative impact on PFS ( $31 \%$ versus $92 \%$ for patients without lymph node involvement; $P<0.001)$ and overall survival ( $80 \%$ versus $100 \%$, respectively; $P=0.02) .{ }^{11}$ Our patients had a 5-year PFS of $72.2 \%$; this is superior to that reported by Thomas et al, ${ }^{11}$ which may be attributable to the small percentage of patients with advanced-stage CCA in our series. Because so few of our patients had recurrences, we were not able to investigate predictors of recurrence.

The prognosis of early-stage CCAs is similar to that of other types of cervical cancer; thus, we considered fertility-preserving treatment a rational treatment option. 
Table 2 Characteristics of patients with recurrent or uncontrolled disease $(n=4)$

\begin{tabular}{llllll}
\hline Patient & Age (years) & Stage & Treatment & High-risk factors & Result (time/recurrence position) \\
\hline I & 39 & IIAI & Chemoradiation & TD $=4 \mathrm{~cm}$ & Uncontrolled $(3 \mathrm{months} /$ lungs $)$ \\
2 & 19 & IIA2 & NAC+RH (LND+RT) (unfinished) & $\begin{array}{l}\text { TD }=I \mathrm{~cm} \\
\text { Deep stromal invasion, PLN(+) }\end{array}$ & Rec (3I months/liver+PLN+PALN) \\
3 & 38 & IBI & RH & No residual disease* & $\begin{array}{l}\text { Rec (I5 months/PALN+ } \\
\text { peritoneum) }\end{array}$ \\
4 & 73 & IIB & Chemoradiation & TD $\geq 4 \mathrm{~cm}$ & Dead (4 months/lungs) \\
\hline
\end{tabular}

Note: *After preoperative diagnostic cone biopsy.

Abbreviations: n, number; TD, tumor diameter; NAC, neoadjuvant chemotherapy; RH, radical hysterectomy; LND, lymphadenectomy; RT, radiation therapy; PLN, pelvic lymph node; rec, recurrence; PALN, para-aortic lymph node.

No guidelines have been established for the treatment of CCAs, as their treatment is mainly based on experience with SCCs and adenocarcinomas. National Comprehensive Cancer Network guidelines recommend cervical cold-knife conization or radical trachelectomy (abdominal or vaginal) for early-stage cervical cancer. ${ }^{19}$ Several case reports of fertility-preserving treatment of CCAs have been published. In one such case report, a 13-year-old patient with stage IIB1 CCA who was treated with neoadjuvant chemotherapy, laparoscopic pelvic lymphadenectomy, vaginal radical trachelectomy, and adjuvant chemotherapy remained disease free after 14 months of followup. ${ }^{13}$ Because there are too few of these cases for a multivariate analysis, whether fertility-preserving treatment for early-stage adenocarcinomas is associated with a worse prognosis than SCCs is still controversial. Several large published studies have reported comparable survival rates for different histological types of cervical cancer, whereas others have drawn different conclusions.$^{20}$ Differing proportions of various high-risk factors may contribute to the discrepancies among these reports. In our study, three patients with neoplasms of diameter less than $2 \mathrm{~cm}$ and no other high-risk factors underwent fertilitypreserving surgery and remained disease free after 8 months, 16 months, and 54 months. Although our data support the feasibility of fertility-preserving treatment in patients with early-stage CCA, because of the high prevalence of infiltration of the cervical canal it is mandatory to employ magnetic resonance imaging to preoperatively assess tumor size and location, degree of extension into and length of the endocervical canal, and the distance between the upper margin of the lesion and the isthmus.

Our study had some shortcomings. The small number of patients with recurrences did not allow the analysis of predictors of recurrence. In addition, the small proportion of patients with advanced-stage CCAs prevented us from evaluating their prognosis.

In conclusion, CCAs may affect adolescent and young patients without prior DES exposure. Diagnosis is often delayed in these patients because of nonspecificity of symptoms, refusal of gynecological examination, and falsenegative results on Pap tests. Of itself, clear cell histology does not appear to portend a worse prognosis than SCC of the cervix in the absence of known risk factors. It is possible to preserve fertility in selected young patients.

\section{Disclosure}

The authors report no conflicts of interest in this work.

\section{References}

1. Reich O, Tamussino K, Lahousen M, Pickel H, Haas J, Winter R. Clear cell carcinoma of the uterine cervix: pathology and prognosis in surgically treated stage IB-IIB disease in women not exposed in utero to diethylstilbestrol. Gynecol Oncol. 2000;76(3):331-335.

2. Garg MM, Arora VK. Clear cell adenosquamous carcinoma of the cervix: a case report with discussion of the differential diagnosis. Int $J$ Gynecol Pathol. 2012;31(3):294-296.

3. Herbst AL, Ulfelder H, Poskanzer DC, Longo LD. Adenocarcinoma of the vagina. Association of maternal stilbestrol therapy with tumor appearance in young women. 1971. Am J Obstet Gynecol. 1999;181(6): 1574-1575.

4. Sassoon D. Wnt genes and endocrine disruption of the female reproductive tract: a genetic approach. Mol Cell Endocrinol. 1999;158(1-2): $1-5$.

5. Troisi R, Hatch EE, Titus-Ernstoff L, et al. Cancer risk in women prenatally exposed to diethylstilbestrol. Int J Cancer. 2007;121(2): 356-360.

6. Hiromura T, Tanaka YO, Nishioka T, Satoh M, Tomita K. Clear cell adenocarcinoma of the uterine cervix arising from a background of cervical endometriosis. Br J Radiol. 2009;82(973):e20-e22.

7. Kocken M, Baalbergen A, Snijders PJ, et al. High-risk human papillomavirus seems not involved in DES-related and of limited importance in nonDES related clear-cell carcinoma of the cervix. Gynecol Oncol. 2011;122(2):297-302.

8. Goto K, Takeuchi Y, Yakihara A, Kotsuji F. Synchronous invasive squamous cell carcinoma and clear cell adenocarcinoma of the uterine cervix: a different human papillomavirus status. Gynecol Oncol. 2005;97(3):976-979.

9. Pirog EC, Kleter B, Olgac S, et al. Prevalence of human papillomavirus DNA in different histological subtypes of cervical adenocarcinoma. Am J Pathol. 2000;157(4):1055-1062.

10. Ueno S, Sudo T, Oka N, et al. Absence of human papillomavirus infection and activation of PI3K-AKT pathway in cervical clear cell carcinoma. Int J Gynecol Cancer. 2013;23(6):1084-1091.

11. Thomas MB, Wright JD, Leiser AL, et al. Clear cell carcinoma of the cervix: a multi-institutional review in the post-DES era. Gynecol Oncol. 2008;109(3):335-339. 
12. Hanselaar A, van Loosbroek M, Schuurbiers O, Helmerhorst T, Bulten J, Bernhelm J. Clear cell adenocarcinoma of the vagina and cervix. An update of the central Netherlands registry showing twin age incidence peaks. Cancer. 1997;79(11):2229-2236.

13. Singh P, Nicklin J, Hassall T. Neoadjuvant chemotherapy followed by radical vaginal trachelectomy and adjuvant chemotherapy for clear cell cancer of the cervix: a feasible approach and review. Int $J$ Gynecol Cancer. 2011;21(1):137-140.

14. Pecorelli S. Revised FIGO staging for carcinoma of the vulva, cervix, and endometrium. Int J Gynaecol Obstet. 2009;105(2):103-104.

15. McNall RY, Nowicki PD, Miller B, Billups CA, Liu T, Daw NC. Adenocarcinoma of the cervix and vagina in pediatric patients. Pediatr Blood Cancer. 2004;43(3):289-294.

16. Baalbergen A, Veenstra Y, Stalpers LL, Ansink AC. Primary surgery versus primary radiation therapy with or without chemotherapy for early adenocarcinoma of the uterine cervix. Cochrane Database Syst Rev. 2010:CD006248.
17. Rotman M, Sedlis A, Piedmonte MR, et al. A phase III randomized trial of postoperative pelvic irradiation in Stage IB cervical carcinoma with poor prognostic features: follow-up of a gynecologic oncology group study. Int J Radiat Oncol Biol Phys. 2006;65(1):169-176.

18. Galic V, Herzog TJ, Lewin SN, et al. Prognostic significance of adenocarcinoma histology in women with cervical cancer. Gynecol Oncol. 2012;125(2):287-291.

19. Koh WJ, Greer BE, Abu-Rustum NR, et al; National Comprehensive Cancer Network. Cervical cancer. J Natl Compr Canc Netw. 2013;11(3): 320-343.

20. Helpman L, Grisaru D, Covens A. Early adenocarcinoma of the cervix: is radical vaginal trachelectomy safe? Gynecol Oncol. 2011;123(1): 95-98.
OncoTargets and Therapy

\section{Publish your work in this journal}

OncoTargets and Therapy is an international, peer-reviewed, open access journal focusing on the pathological basis of all cancers, potential targets for therapy and treatment protocols employed to improve the management of cancer patients. The journal also focuses on the impact of management programs and new therapeutic agents and protocols on

\section{Dovepress}

patient perspectives such as quality of life, adherence and satisfaction. The manuscript management system is completely online and includes a very quick and fair peer-review system, which is all easy to use. Visit http://www.dovepress.com/testimonials.php to read real quotes from published authors. 